\title{
Identification of neural cells activated by mating stimulus in the periaqueductal gray in female rats
}

\author{
Shunji Yamada and Mitsuhiro Kawata * \\ Department of Anatomy and Neurobiology, Kyoto Prefectural University of Medicine, Kyoto, Japan
}

\section{Edited by:}

Ishwar Parhar, Monash University,

Malaysia

Reviewed by:

Lan Ma, Fudan University, China Toshiya Funabashi, St. Marianna

University School of Medicine,

Japan

*Correspondence:

Mitsuhiro Kawata, Department of Anatomy and Neurobiology, Kyoto Prefectural University of Medicine, Kawaramachi-Hirokoji, Kamigyo-ku, Kyoto 602-8566, Japan

e-mail:mkawata@koto.kpu-m.ac.jp
Induction of lordosis as typical female sexual behavior in rodents is dependent on a mount stimulus from males and blood levels of estrogen. Periaqueductal gray (PAG) efferent neurons have been suggested to be important for lordosis behavior; however, the neurochemical basis remains to be understood. In this study, we neuroanatomically examined (1) whether PAG neurons activated by mating stimulus project to the medullary reticular formation (MRF), which is also a required area for lordosis; and (2) whether these neurons are glutamatergic. Mating stimulus significantly increased the number of cFos-immunoreactive (ir) neurons in the PAG, particularly in its lateral region. Half of cFos-ir neurons in the lateral PAG were positive for a retrograde tracer (FluoroGold; FG) injected into the MRF. cFos-ir neurons also colocalized with mRNA of vesicular glutamate transporter 2 (VGLUT2), a molecular marker for glutamatergic neurons. Using retrograde tracing and in situ hybridization in conjunction with fluorescent microscopy, we also found FG and $v$ GLUT2 mRNA double-positive neurons in the lateral PAG. These results suggest that glutamatergic neurons in the lateral PAG project to the MRF and are involved in lordosis behavior in female rats.

Keywords: periaqueductal gray, cFos, mating stimulus, vesicular glutamate transporter 2, FluoroGold, lordosis

\section{INTRODUCTION}

Lordosis is a typical sexual behavior of a female rodent and is induced by a male mount stimulus under proestrus levels of estrogen. The mount stimulus passes through the anterolateral column of the spinal cord and then inputs into the medullary reticular formation (MRF) and periaqueductal gray (PAG) (Pfaff, 1980). Daniels et al. demonstrated an efferent pathway for lordosis behavior using a pseudorabies virus (PRV) as a transneuronal retrograde tracer (Daniels et al., 1999). When PRV was injected into the lumbar epaxial muscles, which produce a lordosis posture in female rats, the PRV was sequentially labeled in the MRF, PAG, and ventromedial nucleus of the hypothalamus (VMH). The VMH is the main site of action of estrogen for inducing lordosis (Rubin and Barfield, 1983) and estrogen receptor-expressing neurons in this nucleus project to the PAG (Calizo and FlanaganCato, 2003). Thus, the PAG and MRF are important relay areas that reflexively change a male mount stimulus into an output for lordosis posture (Pfaff, 1980).

Electrical stimulation of the PAG induces lordosis behavior (Sakuma and Pfaff, 1979a). Conversely, lesions of the PAG (Sakuma and Pfaff, 1979b) or local lesions in the caudal ventrolateral PAG (Lonstein and Stern, 1998) reduce lordosis in female rats. Manual vaginocervical stimulation (VCS), which induces lordosis, is increased cFos expression in the PAG (Pfaus et al., 1996). Neural connections of the PAG to the MRF are involved in induction of an electromyogram (EMG) response in muscles regulating lordosis in female rats (Robbins et al., 1990). These results suggest that PAG efferent neurons activated by a mating stimulus may be related to induction of lordosis, but the neurotransmitter in PAG neurons projecting to the MRF remains to be understood.

Many reports have shown involvement of glutamate and its receptor in lordosis. Intracerebroventricular (icv) administration of N-methyl-D-aspartic-acid (NMDA), an agonist of the glutamate NMDA receptor, facilitated lordosis in ovariectomized (OVX) rats treated with low-dose estrogen (Gargiulo and Donoso, 1995), and activation of lordosis induced by progesterone in estrogen-treated OVX rats was blocked by icv injection of a NMDA antagonist (Gargiulo et al., 1992). A mRNA for vesicular glutamate transporter 2 (vGLUT2), a molecular marker for glutamatergic neurons (Ziegler et al., 2002, 2012), is expressed in the lateral part of the PAG (Oka et al., 2008). Therefore, we hypothesized that the lateral PAG neurons projecting to the MRF are glutamatergic neurons and that these neurons are involved in lordosis. To investigate this hypothesis, we used neuroanatomical methods to examine (1) whether lateral PAG neurons activated by a mating stimulus directly project to the MRF, and (2) whether these neurons are glutamatergic in estrogen-treated OVX rats.

\section{MATERIALS AND METHODS ANIMALS AND TREATMENTS}

Wistar female rats aged 8 weeks were purchased from Shimizu Laboratory Supplies Co. (Kyoto, Japan) and housed under a 12-h reverse light/dark cycle with free access to food and water. After two consecutive estrus cycles, rats were bilaterally ovariectomized and silastic tubing ( $1.5 \mathrm{~mm}$ i.d.; $3.0 \mathrm{~mm}$ o.d.; $25 \mathrm{~mm}$ length; Dow Corning, Midland, MI) containing crystalline $17 \beta$-estradiol (E2, 
Nachalai, Osaka, Japan) was implanted subcutaneously under anesthesia with 2-3\% isoflurane. We confirmed that the E2 treatment caused hypertrophy of the uterus and induced a high lordosis quotient (>90) against male mount behavior. All experimental procedures were authorized by the Committee for Animal Research, Kyoto Prefectural University of Medicine.

\section{SEXUAL STIMULATION AND TISSUE PREPARATION}

One week after OVX and E2 treatment, the rats were assigned randomly to a sexual stimulus condition. Some female rats were placed into a test arena $(60 \mathrm{~cm}$ long $\times 30 \mathrm{~cm}$ wide $\times 30 \mathrm{~cm}$ high) with a sexually vigorous male (age $>12$ weeks) for $1 \mathrm{~h}$ for mating stimulus at 17:00, and others were placed into the same arena without a male to serve as non-mating stimulated controls. Mating-stimulated female rats received $>10$ mating stimuli within $15 \mathrm{~min}$. At the conclusion of sexual stimulation, all animals were anesthetized with pentobarbital (Somnopentyl; Kyouritsu Seiyaku, Tokyo, Japan) and perfused with physiological saline followed by $4 \%$ paraformaldehyde in $0.05 \mathrm{M} \mathrm{PB}$. The brain was immediately removed, postfixed with the same fixative overnight at $4^{\circ} \mathrm{C}$, and then kept in $30 \%$ sucrose in $0.05 \mathrm{M} \mathrm{PB}$ at $4^{\circ} \mathrm{C}$. Serial coronal sections $(30 \mu \mathrm{m})$ containing the PAG were obtained using a cryostat (CM 3050 S; Leica, Wetzlar, Germany).

\section{cFos IMMUNOHISTOCHEMISTRY (IHC)}

Every fourth section through the PAG (8 sections, from 7.0 to $8.2 \mathrm{~mm}$ posterior to the bregma in the brain atlas (Paxinos and Watson, 2006)) from mating-stimulated $(n=5)$ and control $(n=5)$ rats was sequentially incubated with $0.3 \% \mathrm{H}_{2} \mathrm{O}_{2}$ in PBS with $0.3 \%$ Triton X-100 for $30 \mathrm{~min}$ and $2 \%$ normal goat serum (NGS) in PBS for $1 \mathrm{~h}$ at room temperature (RT). Sections were then incubated with primary rabbit antiserum against cFos (1:15,000; Ab-5, Calbiochem, Merck, Tokyo, Japan) for $24 \mathrm{~h}$ at RT. Immunoreactive (ir) neurons were visualized with a streptavidin-biotin kit (Nichirei, Tokyo, Japan), followed by $3,3^{\prime}$-diaminobenzidine (DAB) with $2.5 \%$ nickel chloride, as described our previous method (Takanami et al., 2010).

\section{FLUOROGOLD (FG) INJECTION INTO THE MRF AND FG AND cFoS DOUBLE-IHC}

Five days after OVX and E2 treatment, rats $(n=9)$ were stereotaxically implanted with a stainless-steel guide cannula (23-gage; Plastics One, Roanoke, VA) in the MRF with the tip end at $11.4 \mathrm{~mm}$ posterior and $9.0 \mathrm{~mm}$ ventral to the bregma and 0.7 lateral to the midline, according to the brain atlas (Paxinos and Watson, 2006). FluoroGold (FG; Invitrogen, Carlsbad, CA) was dissolved in saline at $2 \%$ and unilaterally injected into the $\mathrm{MRF}$ at a rate of $0.25 \mu \mathrm{l} / \mathrm{min}$ for 2 min using a microsyringe pump through an internal cannula (26 gage). This procedure was performed under anesthesia with pentobarbital $(13 \mathrm{mg} / \mathrm{ml}$ Somnopentyl, $0.15 \mathrm{ml} / 100 \mathrm{~g}$ body weight). Two days after FG injection, some rats $(n=6)$ received sexual stimulation and others $(n=3)$ were used as non-stimulated controls. Brains were processed for FG and cFos double-IHC. After cFos-ir was detected as described above, free-floating sections were sequentially incubated with $0.3 \% \mathrm{H}_{2} \mathrm{O}_{2}$ in PBS for $15 \mathrm{~min}, 2 \%$ NGS in PBS for $1 \mathrm{~h}$, and primary rabbit antiserum against
FG (1:20,000; Invitrogen) for $24 \mathrm{~h}$ at RT. FG-ir neurons were visualized with a streptavidin-biotin kit, followed by DAB as a chromogen.

\section{vGLUT2 mRNA IN SITU HYBRIDIZATION (ISH) AND cFos IHC}

To detect $v$ GLUT2 mRNA, cDNA for vGLUT2 (734 bp) was generated by RT-PCR from total RNA of rat hypothalamus. Primers were based on the sequence of rat vGLUT2 (accession number AF271235). The upstream and downstream primers were 5'-CTT CTT GGT GCT TGC AGT GG and 5'-GGA CGA ATG GCC TGA ATG GA, respectively (Ziegler et al., 2002). Non-radioactive free-floating ISH was performed as described previously (Yamada et al., 2007, 2012). Briefly, every fourth section containing the PAG ( 8 sections, $n=$ 6) was acetylated and then hybridized with $2 \mathrm{mg} / \mathrm{ml}$ DIGlabeled $v$ GLUT2 antisense cRNA probes synthesized from cDNA of vGLUT2 using a DIG-labeling kit (Boehringer Mannheim $\mathrm{GmbH}$, Mannheim, Germany) overnight at $55^{\circ} \mathrm{C}$. After elimination of excess cRNA probes, the sections were incubated with $1.5 \%$ blocking reagent (Boehringer Mannheim) and then with an alkaline phosphatase (AP)-conjugated anti-DIG antibody (1:1000, Roche Diagnostics Corp., Indianapolis, IN) for $2 \mathrm{~h}$ at $37^{\circ} \mathrm{C}$. $v G L U T 2$-positive neurons were visualized with a BCIP/NBT solution (1:50, Roche Diagnostics Corp.). After $v G L U T 2$ ISH, cFos IHC was performed as described above.

\section{FLUORESCENT $v$ GLUT2 $\boldsymbol{m R N A}$ ISH AND FG IHC}

Preparation of PAG sections $(n=3)$ after FG injection into the MRF and the procedure until blocking with $1.5 \%$ blocking solution is described in the section on ISH for $v G L U T 2$ mRNA. After blocking, the sections were incubated with a mixture of sheep horseradish peroxidase-conjugated anti-DIG antibody (1:20, Roche Diagnostics Corp.) and rabbit anti-FG antibody (1:1000, Invitrogen) overnight at RT. Then the sections were incubated for $30 \mathrm{~min}$ in biotin-conjugated tyramide (1:50 in amplification diluent, PerkinElmer, Waltham, MA). Following several washings, the sections were incubated with a mixture of Alexa 488-conjugated streptavidin and Alexa 546-conjugated anti-rabbit IgG (1:500, Molecular Probes, Eugene, OR) for $2 \mathrm{~h}$ at RT.

\section{ANALYSIS AND STATISTICS}

After staining, the sections were mounted on APS-coated glass slides and covered with a glass micro-cover slip. Non-fluorescent staining was observed under a light microscope (BX 50; Olympus) and photographs of ipsilateral PAG were captured using a CCD camera (DP 21; Olympus). A frame of size of $0.5 \times 0.5 \mathrm{~mm}$ (region of interest, ROI) was made in the captured lateral PAG and the numbers of cFos-ir, FG-ir, vGLUT2 mRNA-positive, FG-ir and cFos-ir, and vGLUT2 mRNA-positive and cFos-ir neurons in the ROI were counted. Immunofluorescent staining was viewed and captured using a LSM510META confocal laser-scanning microscope (Carl Zeiss, Jena, Germany). All values are expressed as means \pm SEM. The significance of a difference between mating-stimulated and non-stimulated control rats was evaluated by Student $t$-test. 


\section{RESULTS}

\section{ACTIVATION OF LATERAL PAG NEURONS BY MATING STIMULUS}

In non-mating stimulated control rats, which were placed in the test arena without male rats, there were few cFos-ir neurons in the PAG (Figure 1A). In contrast, in mating-stimulated rats, many cFos-ir neurons were present in the rostral to caudal parts of the PAG, particularly in the lateral area (Figure 1B). The number of cFos-ir neurons in the lateral PAG in these rats was fourfold greater than that in control rats $(P<0.05$, Figure 1C).

\section{PROJECTION OF MATING-STIMULATED cFos-EXPRESSING NEURONS IN THE LATERAL PAG}

To investigate whether cFos-expressing lateral PAG neurons induced by a mating stimulus project to the MRF, FG was injected into the MRF of female rats prior to mating stimulus. The injection of FG extended through the reticular formation (RF), including the MRF, and caudal pontine RF (PRF), with a longitudinal distance from 11.28 to $12.48 \mathrm{~mm}$ posterior to the bregma in the brain atlas (Paxinos and Watson, 2006) (Figure 2A). This area included the gigantocellular reticular nucleus (Gi), the gigantocellular reticular nucleus ventral $(\mathrm{GiV})$ and alpha $(\mathrm{GiA})$ regions, and the lateral paragigantocellular nucleus, in which neurons found neuroanatomically (Daniels et al., 1999) and electrophysiologically (Sakuma and Pfaff, 1980) have been suggested to be involved in lordosis. Many FG-ir neurons were distributed bilaterally with an ipsilateral dominance through the rostral to caudal regions of the lateral PAG (Figure 2B). In non-stimulated rats, there were a few cFos-ir and FG and cFos double-ir neurons in the lateral PAG (Figure 2C). In contrast, many FG and cFos double-ir
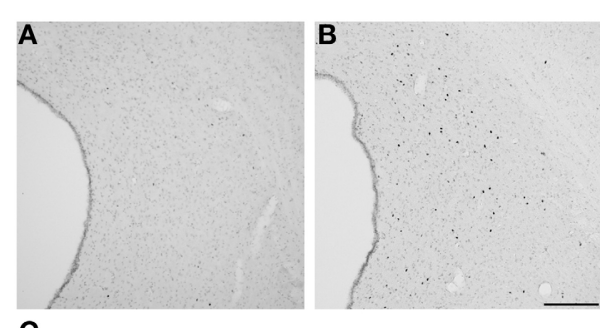

C

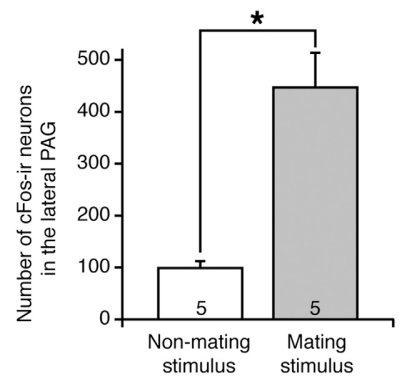

FIGURE 1 | cFos expression in the lateral PAG in representative female rats following (A) non-mating stimulus and (B) mating stimulus. cFos-ir neurons are shown by black dots. (C) The mean number of cFos-ir neurons in the lateral PAG in OVX+E2 rats after mating stimulus (solid bar) was significantly higher than that in non-stimulated control rats (open bar) ( ${ }^{*} P<0.05$; Student $t$-test). Values are shown as means \pm SEM. The numbers in each column indicate the numbers of animals used. Scale bar, $100 \mu \mathrm{m}$. neurons were found in the lateral PAG in mating-stimulated rats (Figure 2D). The numbers of FG and cFos-ir, cFos-ir, and FGir neurons in the ROI $(0.5 \times 0.5 \mathrm{~mm})$ in the lateral PAG were $114.3 \pm 7.9,229.3 \pm 19.7$, and $644.5 \pm 22.2$, respectively. The percentage of FG-ir neurons among total cFos-ir neurons was $50.4 \pm 1.7 \%$ and that of cFos-ir neurons among total FG-ir neurons was $17.9 \pm 1.5 \%$ (Table 1 ). These numbers and percentages were significantly higher $(P<0.05)$ in mating-stimulated rats than in non-stimulated rats, except for FG-ir neurons (Table 1).

\section{NEUROCHEMICAL IDENTITY OF MATING-STIMULATED cFos-EXPRESSING NEURONS IN THE LATERAL PAG}

We performed double staining for $v$ GLUT2 mRNA ISH and cFos IHC to examine whether the mating stimulus-induced cFos-expressing neurons in the lateral PAG are glutamatergic. Many $v G L U T 2$ mRNA-positive neurons were located in the lateral PAG (Figures 3A,B). The distribution pattern of $v G L U T 2$ mRNA-positive neurons was similar to that in a previous study using another type of $v$ GLUT2 cRNA probe (Oka et al., 2008). Several $v$ GLUT2 mRNA-positive neurons showed cFos-ir in
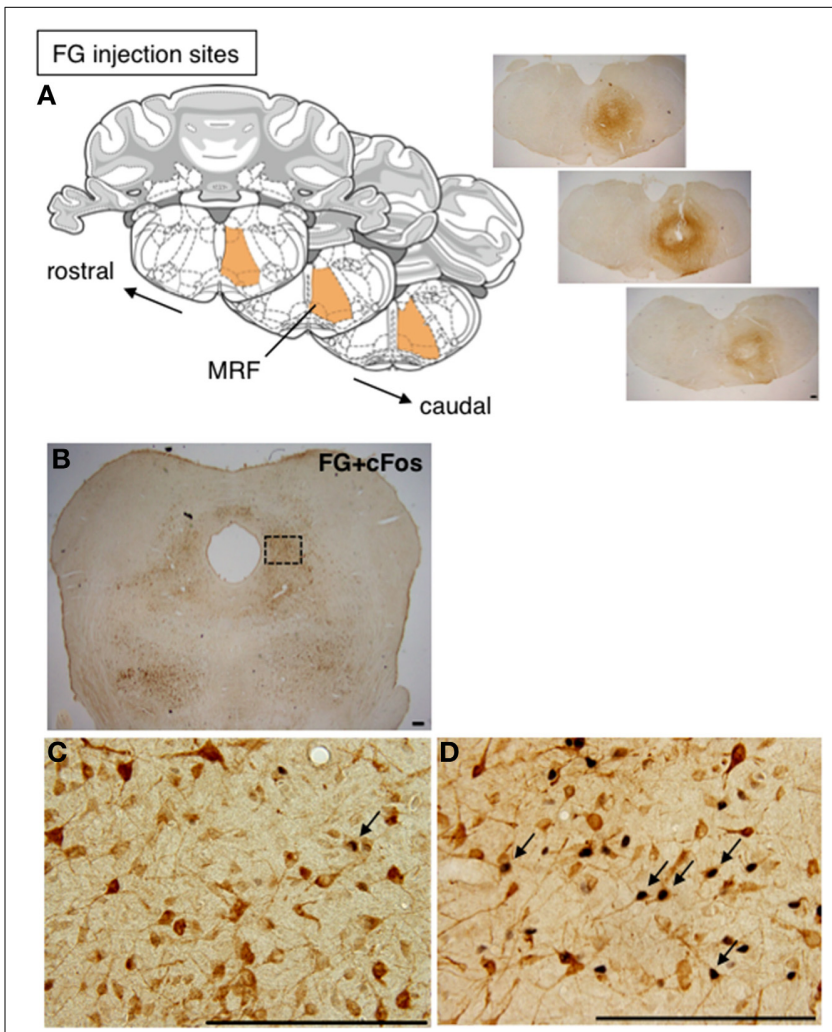

FIGURE 2 | (A) Coronal sections from the rat brain atlas of Paxinos and Watson (2006), showing the position of the MRF and representative photomicrographs showing the site of FG injection in the RF. (B) Representative photomicrographs of the caudal part of the lateral PAG in rat after injection of FG into the RF. High magnification of FG-ir (brown) and cFos-ir (black) neurons in the caudal part of the lateral PAG in a non-stimulated control rat (C) and a mating-stimulated rat (D). Arrows show FG-ir neurons with cFos-ir in nuclei. Scale bar: $200 \mu \mathrm{m}$. 
Table 1 | Numbers and percentages of FG and cFos immunoreactive neurons in the lateral PAG in female rats after FG injection into the RF with or without mating stimulus.

\begin{tabular}{lccccc}
\hline & \multicolumn{3}{c}{ Number of neurons } & & \multicolumn{2}{c}{ Percentages (\%) } \\
\cline { 2 - 5 } & FG and cFos & cFos & FG & FG/cFos & cFos/FG \\
\hline Non-stimulus & $31.0 \pm 3.0$ & $71.7 \pm 8.3$ & $709.7 \pm 41.0$ & $43.5 \pm 1.5$ & $4.4 \pm 1.5$ \\
Mating-stimulus & $114.3 \pm 7.9^{*}$ & $229.3 \pm 19.7^{*}$ & $644.5 \pm 22.2$ & $50.4 \pm 1.7^{*}$ & $17.9 \pm 1.5^{*}$ \\
\hline
\end{tabular}

${ }^{*} P<0.05$ compared with the non-stimulated rats.

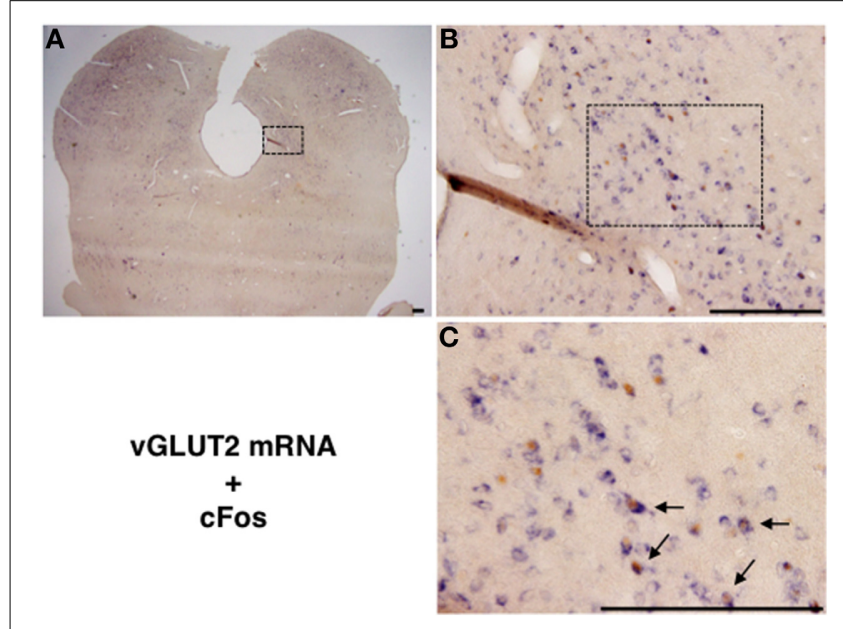

FIGURE 3 | (A) Representative photomicrographs showing vGLUT2 mRNA (purple) in situ hybridization and cFos (brown) immunohistochemistry in the caudal part of the lateral PAG. (B) High magnification of the square in (A). (C) Enlarged view of several neurons in the square in (B). Arrows show vGLUT2 mRNA and Fos-ir neurons. Scale bar: $200 \mu \mathrm{m}$.

nuclei (Figure 3C). The number of $v$ GLUT2 mRNA-positive and cFos-ir neurons, cFos-ir neurons, and $v$ GLUT2 mRNA-positive neurons in the ROI $(0.5 \times 0.5 \mathrm{~mm})$ in the lateral PAG were $74.8 \pm$ $11.1,131.0 \pm 11.5$, and $421.2 \pm 31.0$, respectively. The percentage of $v$ GLUT2 mRNA-positive neurons among total cFos-ir neurons was $55.6 \pm 3.9 \%$ and that of cFos-ir neurons among total $v$ GLUT2 mRNA-positive neurons was $17.4 \pm 1.6 \%$ (Table 2). There were no hybridization signals in brain sections incubated with sense probes for $v$ GLUT2 (data not shown).

\section{PROJECTION OF vGLUT2-POSITIVE NEURONS IN THE LATERAL PAG TO THE MRF}

We investigated whether MRF-projecting lateral PAG neurons were positive for $v G L U T 2$ mRNA using double fluorescence staining for $v$ GLUT2 mRNA ISH and FG IHC with enhancement of ISH signals by biotin-tyramide. Two out of 3 rats received a successful FG injection into the RF. Among FG-ir neurons, 75\% were positive for $v G L U T 2 \mathrm{mRNA}$ in the lateral PAG ipsilateral to the injection site (Figure 4).

\section{DISCUSSION}

The results of the study show that (1) a mating stimulus activates neurons in the lateral PAG, (2) 50\% of lateral PAG neurons activated by the mating stimulus project to the RF, (3) $56 \%$ of these
Table 2 | Numbers and percentages of vGLUT2 mRNA-positive and cFos immunoreactive neurons in the lateral PAG in female rats after mating stimulus.

\begin{tabular}{lccccc}
\hline \multicolumn{3}{c}{ Number of neurons } & \multicolumn{2}{c}{ Percentages $(\%)$} \\
\cline { 1 - 2 } \cline { 5 - 6 } $\begin{array}{l}\text { vGLUT2 and } \\
\text { cFos }\end{array}$ & cFos & vGLUT2 & & $\begin{array}{c}\text { vGLUT2/ } \\
\text { cFos }\end{array}$ & $\begin{array}{c}\text { cFos/ } \\
\text { vGLUT2 }\end{array}$ \\
\hline $74.8 \pm 11.1$ & $131.0 \pm 11.5$ & $421.2 \pm 31.0$ & $55.6 \pm 3.9$ & $17.4 \pm 1.6$ \\
\hline
\end{tabular}

$N=6 ; 8$ sections in each rats.

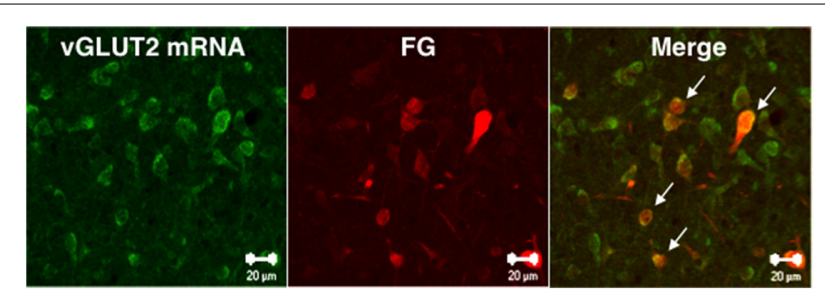

FIGURE 4 | Confocal microscope images showing vGLUT2 mRNA-positive (green) and FG-ir (red) neurons in the caudal part of lateral PAG. Arrows indicate vGLUT2 mRNA-positive and FG-ir double-stained neurons. Scale bars: $20 \mu \mathrm{m}$.

neurons are glutamatergic, and (4) there are glutamatergic neurons projecting to the RF. The PAG and MRF are essential sites for lordosis behavior in female rats (Pfaff, 1980) and there is a clear relationship between glutamate and induction of lordosis (Gargiulo et al., 1992; Gargiulo and Donoso, 1995; Landa et al., 2009). Our findings provide further evidence that glutamatergic neurons in the lateral PAG project to the MRF and are involved in lordosis in female rats.

The PAG receives input from many brain areas, including the forebrain, hypothalamus, and brainstem, and has reciprocal efferent neurons linked with these brain areas (Paxinos, 2004). Functionally, the PAG is associated with modulation of pain and defensive behavior, in addition to lordosis (Paxinos, 2004). Thus, although we and others have shown increased cFos expression by VCS of mating or manual probing in the PAG (Tetel et al., 1993; Pfaus et al., 1996), the function of the activated PAG neurons is still not understood. In the current study, half of the matinginduced cFos-expressing neurons in the lateral PAG were found to project to the RF (MRF and PRF) using a retrograde tracer, and the MRF has also been implicated in induction of lordosis. For example, lesions in the MRF disrupt lordosis (Zemlan et al., 1983) 
and electrical stimulation of the MRF causes an EMG response in lordosis-inducing muscles (Femano et al., 1984). Moreover, PAG neurons are antidromically activated by electrical stimulation of the MRF in female rats (Sakuma and Pfaff, 1980). These results suggest that sensory information induced by a mount stimulus has afferent inputs in the lateral PAG and activates neurons projecting to the MRF, after which MRF neurons cause lordosis behavior in female rats.

Contradictory effects of glutamate on lordosis have been found. Icv injection of NMDA accelerated lordosis in low estrogen-primed OVX rats that rarely showed lordosis (Gargiulo and Donoso, 1995; Landa et al., 2009); whereas local injection of NMDA into the VMH inhibited lordosis in estrogen- and progesterone-treated OVX rats that showed frequent lordosis behavior (Georgescu and Pfaus, 2006). In similar rats, subcutaneous injection of MK-801, a NMDA antagonist, inhibited lordosis (Fleischmann et al., 1991), but injection into the ventral tegmental area increased lordosis (Petralia et al., 2007). In another study, an increase in lordosis induced by progesterone and luteinizing hormone-releasing hormone (LHRH) was inhibited by icv administration of a NMDA antagonist (Gargiulo et al., 1992). These results suggest condition- or region-specific effects of glutamate on lordosis behavior.

In the current study, using $v G L U T 2$ in situ hybridization, we first showed activation of lateral PAG glutamatergic neurons by mating stimulus in OVX $+\mathrm{E} 2$ rats, indicating involvement of PAG glutamatergic neurons in lordosis. Double fluorescence for vGLUT2 mRNA ISH and FG IHC showed the presence of lateral PAG glutamatergic neurons projecting to the RF. mRNAs for the NMDA receptor and its subunit are abundant in the MRF (Keifer and Carr, 2000; Matsuda et al., 2002). It is also likely that the NMDA receptor in the MRF is involved in lordosis because activation of lordosis-relevant muscles by electrical stimulation of the MRF was more effective in rats with additional NMDA in the MRF, compared with controls (Robbins et al., 1992). Triple-labeled histological analysis for cFos, FG IHC, and $v$ GLUT2 mRNA ISH was not performed, but we suggest that lateral PAG glutamatergic neurons with axonal connections to the MRF are an important neural pathway for induction of lordosis.

Several lines of evidence suggest that many neurotransmitters are related to regulation of lordosis. Thus, microinfusion of LHRH (Sakuma and Pfaff, 1983), prolactin (Harlan et al., 1983), and substance $P$ (Dornan et al., 1987) into the PAG induces lordosis. Findings for immunoreactive nerve terminals in the PAG (Ljungdahl et al., 1978; Liposits and Setalo, 1980; Harlan et al., 1983) suggest that these peptides may be neurotransmitters or neuromodulaters that convey a mount stimulus from the spinal cord or estrogen information from the VMH to PAG glutamatergic neurons to induce lordosis.

In this study, half of mating-induced cFos-expressing neurons were not FG-ir in the lateral PAG. Some hypothalamic nuclei have an increased number of cFos-expressing neurons after mating stimulus, but not following a manual sensory stimulus of the flank and rump (Pfaus et al., 1993). Sensory stimulation of the flank and rump by a male forefoot during mount behavior is important for lordosis in female rats because denervation of the perineum, tail base, posterior rump and ventral flanks suppresses lordosis (Kow, 1976). The mating stimulus from male rats in the current study included sensory stimuli of the flank and rump and VCS in females, which suggests that the stimulus also induces activation of lateral PAG neurons that are not associated with lordosis. There are afferent projections from the PAG to the thalamus and parabrachial nucleus, which are related to cognition and pain (Sim and Joseph, 1992; Krout et al., 1998). Thus, FG-negative cFos-expressing neurons in the lateral PAG may have a role in modulating the nociceptive mechanism during lordosis. In addition, half of mating-induced cFos-expressing neurons were not glutamatergic in the lateral PAG. GABA- or neurotensin-expressing neural cell bodies are present in the PAG (Paxinos, 2004) and GABA is involved in lordosis behavior (Wada et al., 2008). This indicates that GABA neurons are a candidate for the neurons activated by mating stimulus in the lateral PAG.

We previously showed cFos IHC following ISH using a Kiss 1 DIG-labeling probe (Adachi et al., 2007). In the current study, cFos IHC following $v$ GLUT2 mRNA ISH was performed using the same technique, except for the difference in the DIG-labeling probe. However, cFos expression in the combination of cFos IHC with $v$ GLUT2 ISH was lower than that in cFos and FG double-IHC. This may have occurred because incubation with the $v$ GLUT2 DIG-labeling probe at $55^{\circ} \mathrm{C}$ might have masked an antigenic determinant of cFos for our antibody.

The precise activated area of the MRF in lordosis is not completely clear. We investigated mating stimulus-induced cFos expression in the MRF, but did not detect a cFos signal in this procedure (data not shown). Immediate early genes, including cFos, are sometimes not induced in brain regions containing neurons with spontaneous and high baseline firing rates prior to stimulation of areas such as the MRF (Pfaus and Heeb, 1997). In the current study, the widespread distribution of FG in the RF indicates the presence of mating stimulus-activated glutamatergic neurons in the lateral PAG projecting to the MRF. Our data raise the possibility that MRF neurons distributed around glutamatergic terminals from the lateral PAG can influence lordosis. Further studies are needed to address the glutamatergic influences on these neurons in regulation of lordosis.

\section{ACKNOWLEDGMENTS}

We thank Atsuko Yashiro and Nobunari Tomura for providing experimental support. This work was supported by Grants-in-Aid for Scientific Research from the Ministry of Education, Science, Sports, Culture and Technology, Japan (KAKENHI 24300128 to Mitsuhiro Kawata and 25870621 to Shunji Yamada).

\section{REFERENCES}

Adachi, S., Yamada, S., Takatsu, Y., Matsui, H., Kinoshita, M., Takase, K., et al. (2007). Involvement of anteroventral periventricular metastin/kisspeptin neurons in estrogen positive feedback action on luteinizing hormone release in female rats. J. Reprod. Dev. 53, 367-378. doi: 10.1262/ jrd. 18146

Calizo, L. H., and Flanagan-Cato, L. M. (2003). Hormonal-neural integration in the female rat ventromedial hypothalamus: triple labeling for estrogen receptor-alpha, retrograde tract tracing from the periaqueductal gray, 
and mating-induced Fos expression. Endocrinology 144, 5430-5440. doi: 10.1210/en.2003-0331en.2003-0331

Daniels, D., Miselis, R. R., and Flanagan-Cato, L. M. (1999). Central neuronal circuit innervating the lordosis-producing muscles defined by transneuronal transport of pseudorabies virus. J. Neurosci. 19, 2823-2833.

Dornan, W. A., Malsbury, C. W., and Penney, R. B. (1987). Facilitation of lordosis by injection of substance $\mathrm{P}$ into the midbrain central gray. Neuroendocrinology 45, 498-506. doi: 10.1159/000124781

Femano, P. A., Schwartz-Giblin, S., and Pfaff, D. W. (1984). Brain stem reticular influences on lumbar axial muscle activity. I. effective sites. Am. J. Physiol. 246, R389-R395.

Fleischmann, A., Vincent, P. A., and Etgen, A. M. (1991). Effects of non-competitive NMDA receptor antagonists on reproductive and motor behaviors in female rats. Brain Res. 568, 138-146. doi: 10.1016/0006-8993(91)91389-I

Gargiulo, P. A., and Donoso, A. O. (1995). Interaction between glutamate and luteinizing hormone-releasing hormone (LHRH) in lordosis behavior and luteinizing hormone release (LH): further studies on NMDA receptor mediation. Physiol. Behav. 58, 169-173. doi: 10.1016/0031-9384(95) 00040-P

Gargiulo, P. A., Munoz, V., and Donoso, A. O. (1992). Inhibition by N-methyl$\mathrm{D}$-aspartic acid (NMDA) receptor antagonist of lordosis behavior induced by estrogen followed by progesterone or luteinizing hormone-releasing hormone (LHRH) in the rat. Physiol. Behav. 52, 737-739. doi: 10.1016/00319384(92)90407-S

Georgescu, M., and Pfaus, J. G. (2006). Role of glutamate receptors in the ventromedial hypothalamus in the regulation of female rat sexual behaviors I. Behavioral effects of glutamate and its selective receptor agonists AMPA, NMDA and kainate. Pharmacol. Biochem. Behav. 83, 322-332. doi: 10.1016/j.pbb.2006.02.016

Harlan, R. E., Shivers, B. D., and Pfaff, D. W. (1983). Midbrain microinfusions of prolactin increase the estrogen-dependent behavior, lordosis. Science 219, 1451-1453. doi: 10.1126/science.6828874

Keifer, J., and Carr, M. T. (2000). Immunocytochemical localization of glutamate receptor subunits in the brain stem and cerebellum of the turtle Chrysemys picta. J. Comp. Neurol. 427, 455-468. doi: 10.1002/10969861(20001120)427:3<455::AID-CNE11>3.0.CO;2-X

Kow, L. M. (1976). Sensory requirements for the lordosis reflex in female rats. Brain Res. 101, 47-66. doi: 10.1016/0006-8993(76)90987-2

Krout, K. E., Jansen, A. S., and Loewy, A. D. (1998). Periaqueductal gray matter projection to the parabrachial nucleus in rat. J. Comp. Neurol. 401, 437-454.

Landa, A. I., Gargiulo, A. J., Gargiulo, M. M., Cabrera, R. J., Bregonzio, C., Lafuente Sanchez, J. V., et al. (2009). Alpha and beta noradrenergic mediation of NMDA glutamatergic effects on lordosis behaviour and plasmatic LH concentrations in the primed female rat. J. Neural Transm. 116, 551-557. doi: 10.1007/s00702009-0217-x

Liposits, Z., and Setalo, G. (1980). Descending luteinizing hormone-releasing hormone (LH-RH) nerve fibers to the midbrain of the rat. Neurosci. Lett. 20, 1-4. doi: 10.1016/0304-3940(80)90223-2

Ljungdahl, A., Hokfelt, T., and Nilsson, G. (1978). Distribution of substance P-like immunoreactivity in the central nervous system of the rat-I. Cell bodies and nerve terminals. Neuroscience 3, 861-943. doi: 10.1016/0306-4522(78) 90116-1

Lonstein, J. S., and Stern, J. M. (1998). Site and behavioral specificity of periaqueductal gray lesions on postpartum sexual, maternal, and aggressive behaviors in rats. Brain Res. 804, 21-35. doi: 10.1016/S0006-8993(98) 00642-8

Matsuda, K., Kamiya, Y., Matsuda, S., and Yuzaki, M. (2002). Cloning and characterization of a novel NMDA receptor subunit NR3B: a dominant subunit that reduces calcium permeability. Brain Res. Mol. Brain Res. 100, 43-52. doi: 10.1016/S0169-328X(02)00173-0

Oka, T., Tsumori, T., Yokota, S., and Yasui, Y. (2008). Neuroanatomical and neurochemical organization of projections from the central amygdaloid nucleus to the nucleus retroambiguus via the periaqueductal gray in the rat. Neurosci. Res. 62, 286-298. doi: 10.1016/j.neures.2008.10.004

Paxinos, G. (2004). The Rat Nervous System. San Diego, CA: Gulf Professional Publishing.

Paxinos, G., and Watson, C. (2006). The Rat Brain in Stereotaxic Coordinates. San Diego, CA: Hard Cover Edn. Access Online via Elsevier.
Petralia, S. M., Debold, J. F., and Frye, C. A. (2007). MK-801 infusions to the ventral tegmental area and ventromedial hypothalamus produce opposite effects on lordosis of hormone-primed rats. Pharmacol. Biochem. Behav. 86, 377-385. doi: 10.1016/j.pbb.2007.01.005

Pfaff, D. W. (1980). Estrogens and Brain Function: Neural Analysis of a HormoneControlled Mammalian Reproductive Behavior. New York, NY: Springer-Verlag.

Pfaus, J. G., and Heeb, M. M. (1997). Implications of immediate-early gene induction in the brain following sexual stimulation of female and male rodents. Brain Res. Bull. 44, 397-407. doi: 10.1016/S0361-9230(97) 00219-0

Pfaus, J. G., Kleopoulos, S. P., Mobbs, C. V., Gibbs, R. B., and Pfaff, D. W. (1993). Sexual stimulation activates c-fos within estrogen-concentrating regions of the female rat forebrain. Brain Res. 624, 253-267. doi: 10.1016/00068993(93)90085-2

Pfaus, J. G., Marcangione, C., Smith, W. J., Manitt, C., and Abillamaa, H. (1996). Differential induction of Fos in the female rat brain following different amounts of vaginocervical stimulation: modulation by steroid hormones. Brain Res. 741, 314-330. doi: 10.1016/S0006-8993(96)00985-7

Robbins, A., Pfaff, D. W., and Schwartz-Giblin, S. (1992). Reticulospinal and reticuloreticular pathways for activating the lumbar back muscles in the rat. Exp. Brain Res. 92, 46-58. doi: 10.1007/BF00230382

Robbins, A., Schwartz-Giblin, S., and Pfaff, D. W. (1990). Ascending and descending projections to medullary reticular formation sites which activate deep lumbar back muscles in the rat. Exp. Brain Res. 80, 463-474. doi: 10.1007/BF00227988

Rubin, B. S., and Barfield, R. J. (1983). Induction of estrous behavior in ovariectomized rats by sequential replacement of estrogen and progesterone to the ventromedial hypothalamus. Neuroendocrinology 37, 218-224. doi: $10.1159 / 000123546$

Sakuma, Y., and Pfaff, D. W. (1979a). Facilitation of female reproductive behavior from mesensephalic central gray in the rat. Am. J. Physiol. 237, R278-R284.

Sakuma, Y., and Pfaff, D. W. (1979b). Mesencephalic mechanisms for integration of female reproductive behavior in the rat. Am. J. Physiol. 237, R285-R290.

Sakuma, Y., and Pfaff, D. W. (1980). Covergent effects of lordosis-relevant somatosensory and hypothalamic influences on central gray cells in the rat mesencephalon. Exp. Neurol. 70, 269-281. doi: 10.1016/0014-4886(80) 90026-6

Sakuma, Y., and Pfaff, D. W. (1983). Modulation of the lordosis reflex of female rats by $\mathrm{LHRH}$, its antiserum and analogs in the mesencephalic central gray. Neuroendocrinology 36, 218-224. doi: 10.1159/000123459

Sim, L. J., and Joseph, S. A. (1992). Serotonin and substance P afferents to parafascicular and central medial nuclei. Peptides 13, 171-176. doi: 10.1016/01969781(92)90159-Z

Takanami, K., Sakamoto, H., Matsuda, K., Hosokawa, K., Nishi, M. Prossnitz, E. R., et al. (2010). Expression of G protein-coupled receptor 30 in the spinal somatosensory system. Brain Res. 1310, 17-28. doi: 10.1016/j.brainres.2009.11.004

Tetel, M. J., Getzinger, M. J., and Blaustein, J. D. (1993). Fos expression in the rat brain following vaginal-cervical stimulation by mating and manual probing. J. Neuroendocrinol. 5, 397-404. doi: 10.1111/j.1365-2826.1993.tb00500.x

Wada, S., Yamada, S., and Yamanouchi, K. (2008). Additive inhibition of lordosis by simultaneous treatments with GABA(A) and GABA(B) receptor agonists, muscimol and baclofen, in female rats. Pharmacol. Biochem. Behav. 90, 590-593. doi: 10.1016/j.pbb.2008.04.020

Yamada, S., Uenoyama, Y., Deura, C., Minabe, S., Naniwa, Y., Iwata, K., et al. (2012). Oestrogen-dependent suppression of pulsatile luteinising hormone secretion and kiss 1 mRNA expression in the arcuate nucleus during late lactation in rats. J. Neuroendocrinol. 24, 1234-1242. doi: 10.1111/j.1365-2826.2012. 02330.x

Yamada, S., Uenoyama, Y., Kinoshita, M., Iwata, K., Takase, K., Matsui, H., et al. (2007). Inhibition of metastin (kisspeptin-54)-GPR54 signaling in the arcuate nucleus-median eminence region during lactation in rats. Endocrinology 148, 2226-2232. doi: 10.1210/en.2006-1529

Zemlan, F. P., Kow, L. M., and Pfaff, D. W. (1983). Effect of interruption of bulbospinal pathways on lordosis, posture, and locomotion. Exp. Neurol. 81, 177-194. doi: 10.1016/0014-4886(83)90167-X

Ziegler, D. R., Cullinan, W. E., and Herman, J. P. (2002). Distribution of vesicular glutamate transporter mRNA in rat hypothalamus. J. Comp. Neurol. 448, 217-229. doi: 10.1002/cne.10257 
Ziegler, D. R., Edwards, M. R., Ulrich-Lai, Y. M., Herman, J. P., and Cullinan, W. E. (2012). Brainstem origins of glutamatergic innervation of the rat hypothalamic paraventricular nucleus. J. Comp. Neurol. 520, 2369-2394. doi: 10.1002/cne.23043

Conflict of Interest Statement: The authors declare that the research was conducted in the absence of any commercial or financial relationships that could be construed as a potential conflict of interest.

Received: 27 June 2014; accepted: 02 December 2014; published online: 18 December 2014.
Citation: Yamada S and Kawata M (2014) Identification of neural cells activated by mating stimulus in the periaqueductal gray in female rats. Front. Neurosci. 8:421. doi: 10.3389/fnins.2014.00421

This article was submitted to Neuroendocrine Science, a section of the journal Frontiers in Neuroscience.

Copyright (C) 2014 Yamada and Kawata. This is an open-access article distributed under the terms of the Creative Commons Attribution License (CC BY). The use, distribution or reproduction in other forums is permitted, provided the original author(s) or licensor are credited and that the original publication in this journal is cited, in accordance with accepted academic practice. No use, distribution or reproduction is permitted which does not comply with these terms. 